\title{
Retroperitoneal Splendore-Hoeppli Phenomenon 15 Years after Perforated Appendicitis
}

\author{
Estell J. Williams and E. Patchen Dellinger
}

\begin{abstract}
Background: Splendore-Hoeppli phenomenon is a localized host immunologic reaction to antigens of a variety of infectious and non-infectious agents. It results in nodules of material composed of antigen-antibody complexes, tissue debris, and fibrin. It is believed to be a localized immunological response to an antigen-antibody precipitate related to microbes or inert materials. It is associated worldwide mostly with fungal infections and in the United States with certain bacterial infections including Actinomyces israelii, Nocardia spp., and chronic infections of Staphylococcus spp., Streptococci, Proteus, Pseudomonas, and Escherichia coli. It is observed in various locations including skin, subcutaneous tissue, and conjunctiva causing chronic wounds. Interestingly, it can also be induced by inert materials such as silk sutures. However, the majority of literature and case reports identify association of this reaction with chronic infections.

Case Presentation: A patient developed a chronically draining surgical incision 3 y after a complicated perforated appendectomy. It persisted for $12 \mathrm{y}$ with multiple treatments with intravenous (IV) antibiotics and numerous operative debridements before a chronic surgical incision tract was identified containing particulate material with final pathology revealing Splendore-Hoeppli reaction.

Conclusion: This reaffirms the association of the phenomenon with chronic infection and it is likely that the Splendore-Hoeppli nodules promoted the persistence of this condition for $15 \mathrm{y}$ after laparoscopic appendectomy.
\end{abstract}

$\mathbf{S}^{\mathrm{n}}$ PLENDORE-HOEPPLI SYNDROME is a reaction that is observed in some chronic infections as a production of intensely eosinophilic material around microorganisms or biologically inert substances [1,2]. It has been described in the literature affiliated with certain bacterial infections, most commonly nocardiosis and actinomycosis or fungal infections including sporotrichosis, pityrosporum folliculitis, zygomycosis, candidiasis, aspergillosis, and blastomycosis. It has been described commonly in pathology and ophthalmology literature in relation to mucocutaneous infections and with certain bacterial infections including Actinomyces israelii, Nocardia spp. and chronic infections of Staphylococcus spp., Streptococci, Proteus, Pseudomonas, and Escherichia coli [3]. In addition, Splendore-Hoeppli reaction may be observed with non-infective pathology such as hypereosinophilic syndrome and allergic conjunctival granulomas [2] or pancreatitis-related panniculitis [4]. The Splendore-Hoeppli reaction material comprises antigen-antibody complex, tissue debris, and fibrin. Although the exact nature of this reaction is unknown, it is believed to be a localized immunological response to an antigen-antibody precipitate related to fungi, parasites, bacteria, or inert materials. The characteristic formation of the peri-bacterial or peri-fungal Splendore-Hoeppli reaction probably prevents phagocytosis and intracellular killing of the insulting agent leading to chronicity of infection $[2,3]$. Within the surgical literature it is known to be the result of reactive formation of chronic granulating debris because of retained inert material such as suture material.

\section{Case Report}

A 29-year-old male presented in April 1999 with perforated appendicitis that was complicated by bilateral abscesses

Department of Surgery, University of Washington School of Medicine, Seattle, Washington.

(c) Estell J. Williams and E. Patchen Dellinger 2016; Published by Mary Ann Liebert, Inc. This Open Access article is distributed under the terms of the Creative Commons License (http://creativecommons.org/licenses/by/4.0), which permits unrestricted use, distribution, and reproduction in any medium, provided the original work is properly credited. 
and a scrotal abscess. He had bilateral interventional radiologic (IR) drains placed and a groin incision for scrotal abscess, and was treated with multiple antibiotics and hyperbaric oxygen therapy. Approximately 4 mo later, he underwent appendectomy through a McBurney incision. He recovered well. However, subsequently he began having spontaneous drainage from his right inguinal area and subsequently presented for evaluation at our medical center in October 2002. This was managed with local cauterization with silver nitrate. The draining surgical incision persisted and 8 mo later he had an excision of a superficial abscess in the right inguinal area under local anesthesia. It appeared to heal but 2 mo later he presented again with pain and development of a protruding nodule in the right flank at the site of a previous IR drain from 1999. A computed tomography (CT) scan in August 2003 showed a $6 \times 4 \mathrm{~cm}$ enhancing mass in the right pericolic gutter extending down toward the groin consistent with an abscess. This abscess was described as extraperitoneal, extending from the level of the cecum into the right posterior muscles of the thoracic wall to the anterior right inguinal wall. The patient was hospitalized and had a drain placed into this abscess. Fluid removed from this lesion showed branching gram-positive rods resembling Actinomyces. Cultures were only positive for E. coli resistant to ciprofloxacin and sensitive to sulfamethoxazoletrimethroprim and a variety of other antibiotics. Molecular diagnostic workup on the culture was positive for a Collinsella aerofaciens. This is an anaerobic, non-spore-forming grampositive rod that is found in gastrointestinal (GI) flora and was classified previously as part of the Eubacteria genus. He was seen by an infectious disease consultant. Given the clinical course of his infection, it was believed that this was most consistent with an Actinomyces infection, and he completed 8 wks of penicillin G, 4 million units IV, q6 hours via peripherally inserted central catheter (PICC) line and was then switched to oral amoxicillin, 1gm TID. He was also treated prophylactically with sulfamethoxazole-trimethoprim DS, one tab BID until his drain was removed 4 wks later. He did have some additional spontaneous drainage from a superior nodular lesion, but otherwise, he did well with a CT 2 mo later in January 2004, showing interval improvement of right lower quadrant (RLQ) abnormalities without any evidence of drainable fluid collections. A repeat CT in August 2004 demonstrated residual changes near the cecum, probably representing scar tissue, and the patient's amoxicillin was discontinued at that time.

The patient again returned $31 / 2$ y later in February 2008 with recurrent symptoms and drainage from his right flank where his drain had been previously. Cultures grew $3+E$. coli and 2+ gram-positive rods, which were identified as Propionibacterium by molecular diagnostic methods. He was put on amoxicillin clavulanate $875 \mathrm{mg}$ PO twice a day. At that time, the sinus tract on the right flank drained persistently purulent material on a daily basis. It would open and close over a matter of weeks, but mostly remain open. He had no systemic symptoms such as fevers, night sweats, weight loss, or any abdominal pain, diarrhea, or blood in stools. He underwent excision of an external portion of an inflammatory mass and draining sinus in his right flank for diagnostic purposes to rule out Actinomyces infection. Only E. coli grew. He continued to have drainage from the surgical incision and in April 2009 he underwent exploratory laparoscopy with mobilization of the right colon and exploration of the

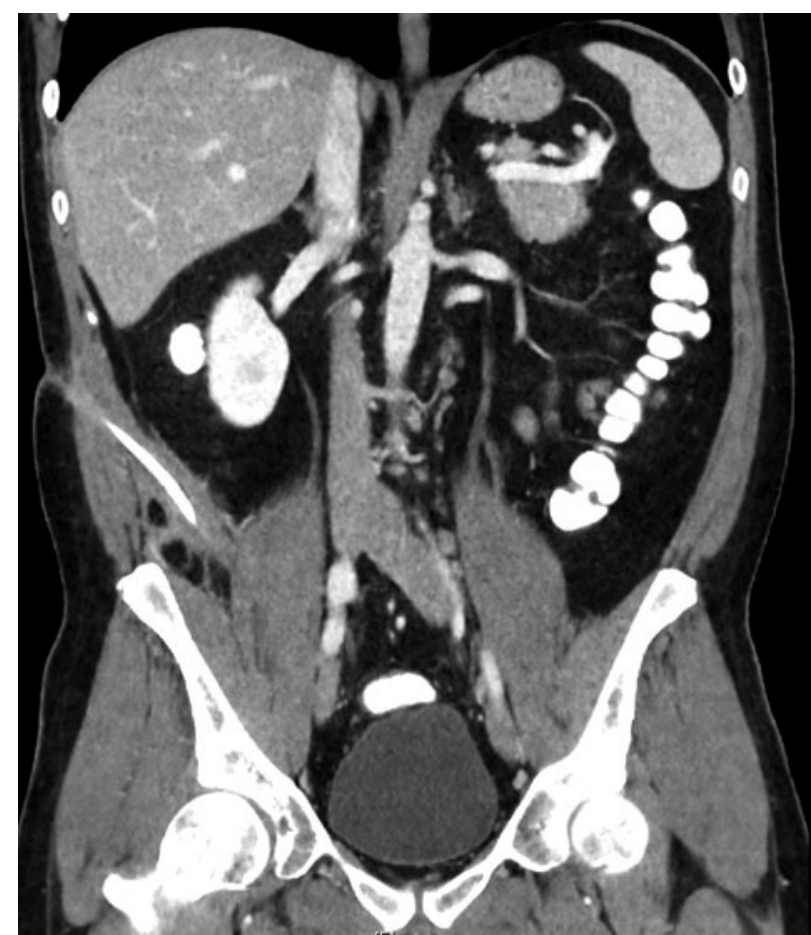

FIG. 1. CT sinogram (August 2013).

retroperitoneum to try to identify the source of his persistently draining right flank sinus. No identifiable tract was found intra-operatively.

His chronically draining surgical incision did not resolve and he presented again $4 \mathrm{y}$ later in August 2013 with the same right flank surgical incision. A CT sinogram was obtained (Fig. 1) and showed a persistent track in the abdominal wall and he was taken to the operating room (OR) and underwent exploratory laparoscopy. A sinogram was obtained in the OR

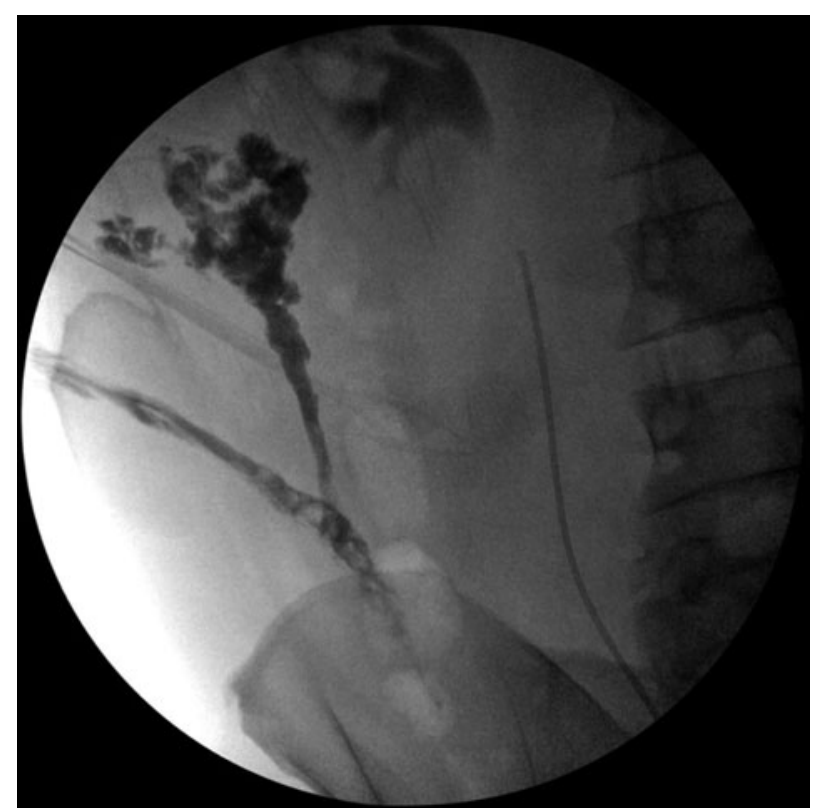

FIG. 2. Operating room sinogram (August 2013). 
(Fig. 2) during the procedure that demonstrated a long sinus tract in the RLQ medial to the iliac wing. The chronic draining sinus was opened exposing what appeared to be fecal material found at end of the tract in the RLQ. The tract also extended cephalad up to the tip of the twelfth rib, but was not opened for the entire distance because of the thick musculature in this region. The infected foreign bodies, believed to be fecoliths, were removed from the retroperitoneum. Pathology examination of these bodies revealed that they exhibited the Splendore-Hoeppli phenomenon.

Following this procedure the drainage improved but recurred again a year and a half later. He developed a tender lump near the tip of his twelfth rib, which is where the images from 2013 showed that his tract had extended. An ultrasound exam of the area in question showed a complex fluid collection just inferior and deep to the right twelfth rib measuring $6.3 \mathrm{~cm} \times 3.6 \mathrm{~cm} \times 4.6 \mathrm{~cm}$, with two associated tracts, one immediately below the skin surface and another heading inferiorly to connect to the old surgical drain tract. There was a thick rind of tissue encasing the complex fluid. Thus, in April 2015, he was taken back to the OR and underwent incision and drainage of the right flank abscess and had a sinogram of the cavity and tract. Pathology results from the tract excised showed skin and subcutaneous tissues with chronic active inflammation, fibrosis, and granulation tissue consistent with deep-seated abscess. Nodular fragments of proteinaceous material consistent with Splendore-Hoeppli phenomenon were again recovered. Culture of the tissue was positive for $E$. coli. Since this procedure, the patient has had complete resolution of his symptoms and the chronic draining surgical incision has healed.

\section{Discussion}

We report a case of Splendore-Hoeppli phenomenon diagnosed $15 \mathrm{y}$ after appendectomy for perforated appendicitis. Suture materials are foreign bodies that induce and prolong tissue reaction in the surgical incision area. In 1971, Varma et al. [2] showed braided polyglycolic acid sutures induced intense acute reaction, mild chronic inflammation, and appeared to be absorbed more slowly than plain or chromic catgut, and tissue reaction was minimal in the absorption stages. A reaction similar to Splendore-Hoeppli phenomenon was observed around multi-filament silk strands in the chronic stages of implantation of polyglycolic and silk sutures. Interestingly, Splendore-Hoeppli reaction can resemble actinomycotic sulfur granules [3]. Thus, it is likely that although this patient was diagnosed with Acti- nomyces infection he really suffered from this reaction and received unnecessary therapy via PICC line intravenous infusion of penicillin, and earlier identification could have prevented this over treatment.

\section{Author Disclosure Statement}

No competing financial interests exist. No funding was received in support of this article.

\section{References}

1. Rodig SJ, Dorfman DM. Splendore-Hoeppli phenomenon. Arch Pathol Lab Med 2001;125:1515-1516.

2. Varma S, Johnson LW, Ferguson HL, et al. Tissue reaction to suture materials in infected surgical wounds: A histopathologic evaluation. Am J Vet Res 1981;42:563-570.

3. Hussein MR. Mucocutaneous Splendore-Hoeppli phenomenon. J Cutan Pathol 2008;35:979-988.

4. Bush JW, Himes E, Liu D, et al. Splendore-Hoeppli reaction and muscular arteritis in pancreatic panniculitis. J Cutan Pathol 2015;42:77-81.

Address correspondence to:

Dr. E. Patchen Dellinger Department of Surgery University of Washington School of Medicine 1959 NE Pacific Street Seattle, WA 98195-6410

E-mail: patch@uw.edu

\begin{aligned} & \multicolumn{1}{c|}{ Abbreviations Used } \\ $\mathrm{CT} & =$ computed tomography \\ $\mathrm{GI} & =$ gastrointestinal \\ $\mathrm{IR} & =$ interventional radiologic \\ $\mathrm{IV} & =$ intravenous \\ $\mathrm{OR} & =$ operating room \\ $\mathrm{PICC} & =$ peripherally inserted central catheter \\ $\mathrm{RLQ} & =$ right lower quadrant \end{aligned}

Cite this article as: Williams EJ and Dellinger EP (2016) Retroperitoneal splendore-hoeppli phenomenon 15 years after perforated appendicitis, Surgical Infections Case Reports 1:1, 26-28, DOI: 10.1089/crsi.2016.0003. 